\title{
Vortex Motion and Quasiparticle Resistivity in Superconductors at Microwave Frequencies
}

\author{
S. Sarti, C. Amabile, R. Fastampa, M. Giura
}

Dipartimento di Fisica and Unità CNISM

Università "La Sapienza", P. le Aldo Moro 2, 00185 Roma, Italy

\author{
E. Silva And N. Pompeo
}

Dipartimento di Fisica "E. Amaldi" and Unità CNISM

Università Roma Tre, Via della Vasca Navale 84, 00146 Roma, Italy

At nonzero frequencies both the normal and superfluid fractions, as well as moving vortices, contribute to the electrical transport, resulting in intricate expressions for the resistivity. We present an extended study of microwave resistivity data measured on $\mathrm{YBa}_{2} \mathrm{Cu}_{3} \mathrm{O}_{7-\delta}, \mathrm{SmBa}_{2} \mathrm{Cu}_{3} \mathrm{O}_{7-\delta}$, and $\mathrm{MgB}_{2}$ by means of a broad-band technique between 2 and $20 \mathrm{GHz}$ and of a resonant system at $50 \mathrm{GHz}$. We discuss the main experimental fingerprints that allow one to identify the relevance of the different contributions from the measured microwave response as a function of frequency, temperature, and magnetic field. We show that the field-dependent superfluid/quasiparticles dynamics cannot be in general neglected with respect to the vortex motion, albeit its relative importance is different in different materials: in high temperature superconductors vortex motion prevails at high fields, but at low fields a significant superfluid/quasiparticle contribution exists (ascribed to the presence of lines of nodes). In $\mathrm{MgB}_{2}$ the two contributions are comparable even at high fields. In this general frame, we derive from the measurements vortex and superfluid parameters in agreement with theoretical predictions and independent measurements.

PACS numbers: 74.25.Nf, 74.20.De, 74.60.Ge, 74.70.Ad, 74.72.--h

\section{Introduction}

In the mixed state of type II superconductors there are two main sources of the response: moving vortices and quasiparticles. In order to catch the main physical features, we will describe both mechanisms using the most simple available models. Further complications may arise if more detailed models need to be used. 
Vortex motion induced by an alternating force of frequency $\nu$ gives rise to a response given by a real part (dissipation) and, in the presence of pinning, by an imaginary (out-of-phase) part. One of the simplest model for the ac complex vortex resistivity $\rho_{\mathrm{v}}$ gives the following expression $[1,2]$ :

$$
\rho_{\mathrm{v}}=\rho_{\mathrm{v} 1}+\mathrm{i} \rho_{\mathrm{v} 2}=\frac{\Phi_{0} B / \eta}{1+\left(\nu / \nu_{0}\right)^{2}}\left[\epsilon+\left(\nu / \nu_{0}\right)^{2}+\mathrm{i} \frac{\nu}{\nu_{0}}(1-\epsilon)\right],
$$

where $\eta$ is the vortex viscosity per unit length, the field induction $B \approx \mu_{0} H$ in the London limit, $\epsilon$ is a creep factor that ranges from $\epsilon=0$ (no flux creep) to $\epsilon=1$ (free vortex motion), and $\nu_{0}$ is a characteristic frequency which, for $\epsilon=0$, corresponds to the depinning frequency $\nu_{\mathrm{p}}=\kappa_{\mathrm{p}} / 2 \pi \eta$.

The second unavoidable source for the measured microwave resistivity, the conductivity $\sigma$ due to charge carriers, can be described by the so-called "two-fluid model" [3] as due to a superconducting fraction $x_{\mathrm{s}}$ and a "normal" fraction (more appropriately, due to quasiparticle excitations with relaxation time $\left.\tau_{\mathrm{qp}}\right) x_{\mathrm{n}}$. When $\omega / 2 \pi=\nu \ll 1 / \tau_{\mathrm{qp}}$, one writes

$$
\sigma=\sigma_{1}-\mathrm{i} \sigma_{2}=\frac{n e^{2}}{m \omega}\left(\omega \tau_{\mathrm{qp}} x_{\mathrm{n}}-\mathrm{i} x_{\mathrm{s}}\right)=\frac{1}{\mu_{0} \omega}\left(\frac{2}{\delta_{\mathrm{nf}}^{2}}-\mathrm{i} \frac{1}{\lambda^{2}}\right),
$$

where $n$ is the charge carrier density, $m$ is the carrier effective mass, and the last equality defines the normal fluid skin depth $\delta_{\mathrm{nf}}(T, B, \nu)$ and the London penetration depth $\lambda(T, B)$.

Since a vortex is made of (and interacts with) superconducting currents, the calculation of the overall resistivity is not trivial. Taking into account the interactions between moving vortices and macroscopic superfluid/normal currents, one finally gets [1]:

$$
\tilde{\rho}=\rho_{1}+\mathrm{i} \rho_{2}=\frac{1-\mathrm{i} \sigma_{2}(B, T) \rho_{\mathrm{v}}(B, T, \omega)}{\sigma_{1}(B, T, \omega)-\mathrm{i} \sigma_{2}(B, T)} .
$$

When dealing with experiments, it is also useful to introduce the magnetic field induced shift in the complex resistivity, normalized to the normal state: $\Delta \tilde{r}=$ $r_{1}+\mathrm{i} r_{2}=[\tilde{\rho}(B, T, \omega)-\tilde{\rho}(0, T, \omega)] / \rho_{\mathrm{n}}$.

All the parameters involved in Eq. (3) may depend on temperature $T$ and magnetic field $B$. As a result, in the general case the extraction of any physical parameter from the measured microwave resistivity may be extremely complex and not always straightforward. In this paper, we show through some examples how the measured microwave complex resistivity can be used to derive physical parameters related to vortex motion and superfluid in the mixed state.

\section{Experimental setup and samples}

All measurements here presented were performed in thin, high-quality superconducting films of $\mathrm{YBa}_{2} \mathrm{Cu}_{3} \mathrm{O}_{7-\delta}$ (YBCO), $\mathrm{SmBa}_{2} \mathrm{Cu}_{3} \mathrm{O}_{7-\delta}$ (SmBCO), and $\mathrm{MgB}_{2}$, with $T_{\mathrm{c}}=89.5 \mathrm{~K}, 87 \mathrm{~K}$, and $36 \mathrm{~K}$, respectively.

The microwave response at high frequency was measured in YBCO and SmBCO by the end-wall cavity technique [4] at $48.2 \mathrm{GHz}$. From the field induced 
change of the quality factor $Q$ and of the resonant frequency $f_{0}$ one obtains the field induced change of the complex resistivity $\Delta \tilde{\rho}(H, T) / d$, since the films had the thickness $d$ sufficiently small to satisfy the so-called thin film approximation [5], hence $\Delta \tilde{r}$.

Frequency dependent measurements were performed in $\mathrm{YBCO}$ and $\mathrm{MgB}_{2}$ films through a Corbino disk geometry [6]: a swept frequency microwave radiation is generated by a vector network analyzer and guided to the sample under study through a coaxial cable. The sample short-circuits the coaxial cable. Measurements of the complex reflection coefficients yielded the complex resistivity under the same thin film approximation as before. Details on samples and experimental setups and procedures can be found in [7] and references therein.

\section{Fixed frequency measurements}

At fixed frequency $\Delta \tilde{\rho}(T, B)$ is determined by the variations of the parameters in Eq. (3). Since all these parameters can vary as a function of $T$ or $B$, in order to extract any information on the vortex motion and/or quasiparticle dissipation some hypotheses must be made. The most used approximation is to consider $\sigma_{1} / \sigma_{2}=\omega \tau_{\mathrm{qp}}\left(1-x_{\mathrm{s}}\right) / x_{\mathrm{s}} \ll 1$ and, at low enough $T$ and $B$, that $x_{s}$ does not depend on $B$ and creep of vortices is negligible $(\epsilon \approx 0)$, so that $\Delta \tilde{\rho}(B, T) \approx \tilde{\rho}_{\mathrm{v}}(B, T)$. In that case, $\nu_{0}$ and $\eta$ are the only relevant parameters, and can be directly extracted from the data. However, in cuprates $\sigma_{1} / \sigma_{2}$ may be not negligible at the high edge of the microwave spectrum [8], and then the field variation of $x_{\mathrm{s}}$ may strongly affect $\Delta \tilde{\rho}(B, T)$. Moreover, on general grounds $x_{\mathrm{s}}(T, B)=x_{\mathrm{s} 0}(T)\left[1-\left(B / B_{\mathrm{c} 2}\right)^{\alpha}\right]$, with $\alpha=\frac{1}{2}$ in a superconductor with lines of nodes [9] (as opposed to $\alpha=1$ in a fully gapped superconductor), which enhances the relevance of $x_{\mathrm{s}}(T, B)$. To clarify this point we report in Fig. 1 some simulated curve on the basis of Eq. (3) with typical parameters, from which it is seen that for large $x_{\mathrm{s} 0}$ (that is, at low temperatures) the response is mainly dictated by the vortex motion, while at higher temperatures (lower values of $x_{\mathrm{s} 0}$ ) the field-induced quasiparticle contribution is relevant and characterized by a pronounced curvature in $\Delta \tilde{\rho}$. Moreover, it is seen that, by including the variation of $x_{\mathrm{s}}$ with $B$ the imaginary part $\Delta r_{2}$ switches from positive to negative with lowering $x_{\mathrm{s}}$. By further analysis of Eq. (3), it can be seen that, for small $B / B_{\mathrm{c} 2}$ and $\nu_{0} / \nu, \Delta \tilde{r} \approx\left[a_{1}(T)+\mathrm{i} a_{2}(T)\right] \sqrt{B}+b_{1}(T) B$, where $a_{1,2}$ are related to superfluid and quasiparticles properties only, and $b_{1} \approx \Phi_{0} / \eta \rho_{\mathrm{n}}$ gives the vortex viscosity. These predicted behaviors can be directly compared to the measured data in RE-BCO films, see Fig. 2. Indeed, the reported values of $\nu_{0}$ for this class of materials are around $10 \mathrm{GHz}$ at our working temperatures [10], so that at our measuring frequency $\nu=48.2 \mathrm{GHz}, \nu_{0} / \nu \approx 0.2$. In the case of $\mathrm{SmBCO}$ a clear downward curvature in $\rho_{1}$ is present at all temperatures, indicating the presence of lines of nodes in the gap and a relevant role of the quasiparticle contribution even far from $T_{\mathrm{c}}$. In the case of $\mathrm{YBCO}$, on the other hand, the downward curvature is present only very close to $T_{\mathrm{c}}$, indicating a smaller relevance of quasiparticles 


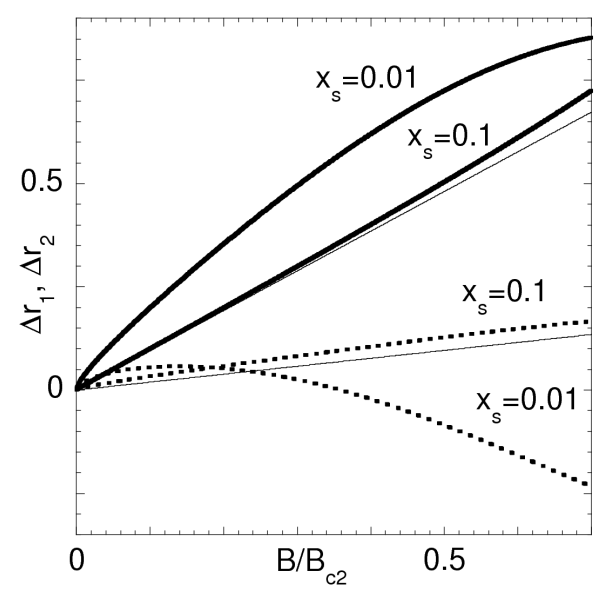

Fig. 1. Normalized complex resistivity shift vs. the normalized magnetic field, calculated by Eq. (3), with $\alpha=\frac{1}{2}$ (lines of nodes), $\nu / \nu_{0}=5, \epsilon=0, \tau_{\mathrm{qp}}=\tau_{\mathrm{n}} \approx 10^{-13} \mathrm{~s}$ and different $x_{\mathrm{s}}$. Continuous lines: $\Delta r_{1}$. Dotted lines: $\Delta r_{2}$. Thin lines refer to vortex motion only (let us note that, as a function of $B / B_{\mathrm{c} 2}$, there is no $x_{\mathrm{s}}$ dependence of the vortex motion contribution).

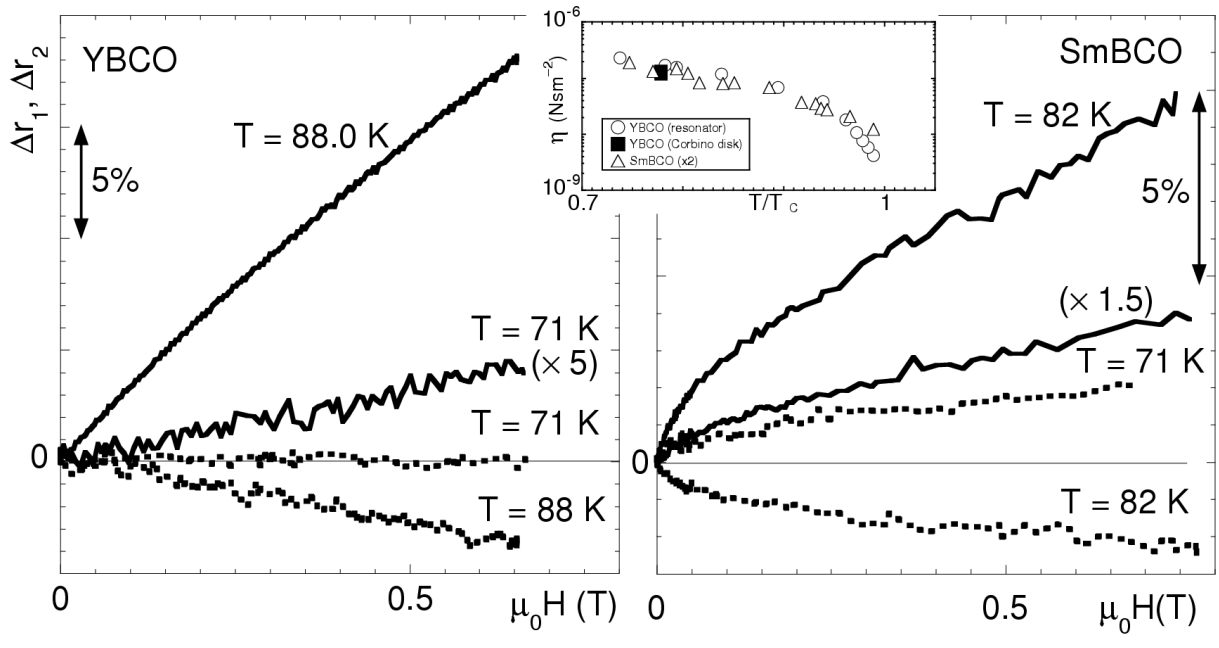

Fig. 2. Normalized complex resistivity shift $\Delta \tilde{r}$ in YBCO (left part) and SmBCO (right part) at different temperatures. Continuous lines: $\Delta r_{1}$. Dotted lines: $\Delta r_{2}$. The curvature in $\Delta \tilde{r}$ and/or the decrease in $\Delta r_{2}$ with $H$ indicate the effect of field-induced superfluid depletion. Inset: vortex viscosity extracted from the measurements. To avoid crowding, some data are multiplied by the factors indicated.

with respect to the case of SmBCO. In both cases, the contribution of the field induced superfluid depletion cannot be neglected, at least in some temperature 
range. On the basis of the previous discussion of the theoretical expectations, we can isolate the quasiparticle/superfluid contribution from the vortex motion term by identifying the $B$-linear term in $\Delta \tilde{r}$ [11]. In this way, we determine $\eta$ for the two cuprate materials under study (inset of Fig. 2). The temperature dependence and absolute values are in agreement with data on crystals [10]. The factor $\approx 2$ between data in SmBCO and YBCO is consistent with the difference in normal state resistivities [7].

\section{Frequency dependent measurements}

When frequency dependent measurements are available, one may make use of Eq. (3) directly. In principle, one could determine all parameters directly from the measured curves. However, this is in most cases impossible, since to this end one would need a very broad spectrum (several decades) and very precise measurements, which are typically not feasible. Some approximations are thus necessary in order to reduce the number of parameters used to fit the measured curves. We here briefly discuss these approximations in the case of $\mathrm{MgB}_{2}$. Due to the two gap nature of this compound, with two coexisting superconducting gaps and correspondingly two interacting superfluids, the simple model depicted in the introduction might be oversimplified. To avoid such complications, we concentrate our interest on the region $\mu_{0} H \gtrsim 1 \mathrm{~T}$, where one of the two superconducting gaps is almost suppressed [12]. Second, since $\mathrm{MgB}_{2}$ is a fully gapped superconductor [13], $x_{\mathrm{s}}$ varies linearly with $B$. This leaves only two field-independent free parameters for the description of the superfluid/quasiparticle contribution $\left(x_{\mathrm{s} 0}(T)\right.$ and $B_{\mathrm{c} 2}(T)$ ). Finally, from Eq. (1) and using $\eta=\Phi_{0} B_{\mathrm{c} 2}(T) / \rho_{\mathrm{n}}$, vortex motion is described by only two additional field and temperature dependent parameters, $\nu_{0}(T, B)$ and $\epsilon(T, B)$. As a result, for each $T$ and $B$ we fit the curves $\rho_{1}(\nu)$ and $\rho_{2}(\nu)$ using only four parameters, two of them depending only on $T$. The entire
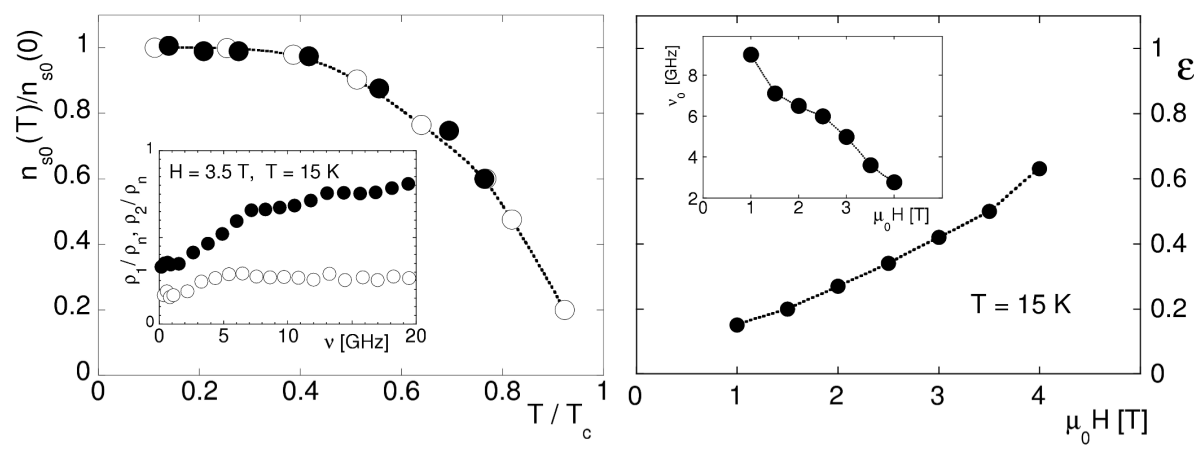

Fig. 3. Corbino disk results on a $\mathrm{MgB}_{2}$ film. Left part: superfluid density $n_{\mathrm{s} 0}$ and comparison with BCS prediction (see the text). Left inset: typical measurements of $\tilde{\rho}$ vs. $\nu$ at fixed temperature and field. Right part: creep factor $\epsilon$ and vortex frequency $\nu_{0}$ (inset) at $T=15 \mathrm{~K}$. 
procedure, that ensures selfconsistency, is described in [12]. Typical data for $\rho_{1}(\nu)$ and $\rho_{2}(\nu)$, together with the obtained parameters, are reported in Fig. 3 . In the left part, we report the superfluid density $x_{\mathrm{s} 0}$ as a function of temperature, compared with data obtained from a pure BCS calculation [3] using experimental data for the temperature dependent superconducting gap [14], without any adjusting factor. As can be seen, the agreement is impressive. $B_{\mathrm{c} 2}$ is found to coincide with the values measured through dc resistivity on the same sample [12]. In the right part, we report the field dependence of the vortex motion parameters, $\nu_{0}$ and $\epsilon$, at an intermediate temperature. As reasonably expected, the creep factor increases with increasing field, indicating an easy escape from pinning wells at denser vortex lattices. The characteristic frequency decreases with field, indicating that pinning wells are steeper at low fields.

\section{Conclusions}

In this paper we have analyzed and discussed different microwave data for the complex resistivity in various superconductors. Using as a basis a very simple model for the microwave response in the mixed state of type-II superconductors, we have shown that in high temperature superconductors and in $\mathrm{MgB}_{2}$ it is in general not possible to neglect the field dependence of the superfluid (and consequently, the creation of quasiparticles). Once this contribution has been recognized, it is possible to obtain information on both the superfluid and vortex motion parameters. As examples, we have illustrated the case of RE-BCO superconductors and $\mathrm{MgB}_{2}$.

\section{Acknowledgments}

We thank C. Ferdeghini and A.M. Cucolo for supplying the $\mathrm{MgB}_{2}$ and RE-BCO samples, respectively.

\section{References}

[1] M.W. Coffey, J.R. Clem, Phys. Rev. Lett. 67, 386 (1991).

[2] E.H. Brandt, Phys. Rev. Lett. 67, 2219 (1991).

[3] M. Tinkham, Introduction to Superconductivity 2nd edition; McGraw-Hill, New York 1996.

[4] E. Silva, A. Lezzerini, M. Lanucara, S. Sarti, R. Marcon, Meas. Sci. Technol. 9, 275 (1998)

[5] S. Sridhar, J. Appl. Phys. 63, 159 (1988); E. Silva, M. Lanucara, R. Marcon, Supercond. Sci. Technol. 9, 934 (1996).

[6] D.H. Wu, J.C. Booth, S.M. Anlage, Phys. Rev. Lett. 75, 525 (1995); S. Sarti, C. Amabile, E. Silva, e-print, cond-mat/0406313 (2004).

[7] E. Silva, N. Pompeo, S. Sarti, C. Amabile, in: Recent Developments in Superconductivity Research, Ed. B.P. Martins, Nova Publishers, New York 2006, condmat/0607676. 
[8] D.A. Bonn, R. Liang, T.M. Riseman, D.J. Baar, D.C. Morgan, K. Zhang, P. Dosanjh, T.L. Duty, A. MacFarlane, G.D. Morris, J.H. Brewer, W.N. Hardy, C. Kallin, A.J. Berlinsky, Phys. Rev. B 47, 11314 (1993).

[9] G.E. Volovik, JETP Lett. 58, 469 (1993); H. Won, K. Maki, Phys. Rev. B 53, 5927 (1996).

[10] Y. Tsuchiya, K. Iwaya, K. Kinoshita, T. Hanaguri, H. Kitano, A. Maeda, K. Shibata, T. Nishizaki, N. Kobayashi, Phys. Rev. B 63, 184517 (2001).

[11] N. Pompeo, L. Muzzi, S. Sarti, R. Marcon, R. Fastampa, M. Giura, M. Boffa, M.C. Cucolo, A.M. Cucolo, C. Camerlingo, E. Silva, J. Phys. Chem. Solids 67, 460 (2006).

[12] S. Sarti, C. Amabile, E. Silva, M. Giura, R. Fastampa, C. Ferdeghini, V. Ferrando, C. Tarantini, Phys. Rev. B 72, 24542 (2005).

[13] P. Seneor, C.-T. Chen, N.-C. Yeh, R.P. Vasquez, L.D. Bell, C.U. Jung, Min-Seok Park, Heon-Jung Kim, W.N. Kang, Sung-Ik Lee, Phys. Rev. B 65, 012505 (2001); J.W. Quilty, S. Lee, A. Yamamoto, S. Tajima, Phys. Rev. Lett. 88, 087001 (2002).

[14] P. Szabó, P. Samuely, J. Kacmarcik, T. Klein, J. Marcus, D. Fruchart, S. Miraglia, C. Marcenat, A.G.M. Jansen, Phys. Rev. Lett. 87, 137005 (2001). 